\title{
BIRMINGHAM UNIVERSITY RADIOCARBON DATES IV
}

\author{
F. W. SHOTTON, D. J. BLUNDELL, and R. E. G. WILLIAMS
}

The University of Birmingham, Birmingham, England

Measurements have continued with both the $1 \mathrm{~L}$ and $6 \mathrm{~L}$ counters. Results are not corrected for $\mathrm{C}^{13}$ fractionation. Errors quoted refer only to the standard deviation calculated from a statistical analysis of sample and background count rates and the Libby half-life of $5570 \pm 30 \mathrm{yr}$. Pretreatment has been continued as described previously (Shotton, Blundell, and Williams, 1969).

\section{SAMPLE DESCRIPTIONS}

I. BRITISH FULL-GLACIAL

Birm-32. Stretton-under-Fosse, Warwickshire

Wood (Ulmus) in Lower Wolston Clay from $15.5 \mathrm{~m}$ depth in Borehole 1285 Midland Connection Motorway near Stretton-under-Fosse, Warwickshire $\left(52^{\circ} 26^{\prime} \mathrm{N}\right.$ Lat, $1^{\circ} 19^{\prime} \mathrm{W}$ Long, Grid. Ref. SP463813). Coll. 1967 and subm. by A. Horton. Comment: measurement helps confirm stratigraphic interpretation.

\section{Birm-74. Four Ashes, Staffordshire}

Plant fragments and twigs from fine gray silt ca. $1 \mathrm{~m}$ depth (Site 20) in Four Ashes Gravel at Four Ashes, Staffordshire $\left(52^{\circ} 40^{\prime} 13^{\prime \prime} \mathrm{N}\right.$ Lat, $2^{\circ} 07^{\prime} 24^{\prime \prime}$ W Long, Grid. Ref. SJ916082). Coll. 1968 and subm. by Anne Morgan. Comment: fauna in sample included Lepidurus and many exclusively $\mathrm{N}$ insect species, indicating cold conditions.

(a) 34,250

Birm-114. Trysull, Staffordshire $-1300$

(b) $>25,000$

(c) $>34,000$

Inner (a) middle (b) and outer (c) fractions from shells (Opercula of Bithynia tentaculata) sieved from calcareous silty clay at ca. $2 \mathrm{~m}$ depth overlying coarse kame gravel and sand at Church Lane Pit, Trysull, Staffordshire (52 $33^{\circ} 0^{\prime \prime}$ N Lat, $2^{\circ} 13^{\prime} 25^{\prime \prime}$ W Long, Grid Ref. SO848946). Coll. 1968 and subm. by A. V. Morgan. Comment: stratigraphy and contained fauna suggest figures are minimum ages. Probably Ipswichian.

$$
47,000+2300
$$

Birm-157. Farm Wood Quarry, Chelford, Cheshire 45,050 в.c.

Wood (Pinus sylvestris) from main organic horizon at $10 \mathrm{~m}$ depth in quarry sec. in Chelford Sands formation at Farm Wood Quarry, Chel- 
ford, Cheshire $\left(53^{\circ} 15^{\prime} \mathrm{N}\right.$ Lat, $2^{\circ} 17^{\prime} \mathrm{W}$ Long, Grid Ref. SJ812731). Coll. 1967 and subm. by P. Worsley. Comment: sample from deposit previously dated at $>52,000$ (GrN-1292) and subsequently by isotopic enrichment at 60,800 \pm 1500 (GrN-1475) (Vogel and Zagwijn, 1967). Deposit believed to have unique and critical position in Early Devensian (Weichselian) of England and to be equivalent to Brörup Interstadial (Simpson and West, 1958; Worsley, 1967; Evans, 1968, p. 213). Sample subm. by Worsley to Hannover lab gave values (Hv-1978, 32,850 \pm 480, unpub.) and (Hv-1979b, 26,200 \pm 390 , unpub.) for humate extract. Birmingham date done on another piece of same trunk subm. to Hannover, measured after 4 successive $\mathrm{NaOH}$ treatments to remove possible contamination. Counter reading of activity slightly exceeded $4 \sigma$ after atmosph. pressure correction. If this experimentally obtained coefficient is only slightly inaccurate, result might have been more correctly expressed as $>47,000$. General conclusion is that Hannover date is too young, as result of contamination, and that there is no case for substantial alteration of Groningen figures.

\section{Birm-113. Thrapston, Huntingdonshire}

Twigs from organic-silt lens containing mature tundra assemblage of coleoptera ca. $5 \mathrm{~m}$ depth in terrace gravels of $\mathrm{R}$. Nene, Thrapston, Huntingdonshire $\left(52^{\circ} 24^{\prime} 40^{\prime \prime} \mathrm{N}\right.$ Lat, $0^{\circ} 32^{\prime} 50^{\prime \prime}$ W Long, Grid Ref. JP988805). Coll. 1967 and subm. by G. R. Coope.

(a) 36,300

\section{Birm-161. Scandal Beck, Westmorland}

Sample after alkali pretreatment (a) and humate extract (b) from peat from lower of 2 organic horizons in sandy silt overlain by $1.5 \mathrm{~m}$ till at ca. $3 \mathrm{~m}$ depth on W bank Scandal Beck, $64 \mathrm{~m}$ SSW Brunt Hill Farm, Ravenstonedale, Westmorland (54 $25^{\circ}$ N Lat, $2^{\circ} 24^{\prime}$ W Long, Grid Ref. NY743024). Coll. 1969 and subm. by G. A. L. Johnson. Comment: indicates late Devensian (Weichselian) till upon deposits of Upton Warren interstadial.

\section{Birm-93. Kilmaurs, Ayrshire}

Collagen fraction from antler of Rangifer tarandus from gravel ca. $12 \mathrm{~m}$ deep below till $5 \mathrm{~m}$ thick (part of V 5187, Fig. 1b, p. 4, Gregory and Currie, 1928) at Woodhill Quarry, Kilmaurs, Ayrshire (55 $38^{\prime} \mathrm{N}$ Lat, $4^{\circ} 32^{\prime}$ W Long, Grid Ref. NS410410). Coll. 1865 by J. Bryce; subm. by W. D. Rolfe and W. W. Bishop. Comment: although long stored in 
museum, antler was free from preservative. Date contrasts with 13,700 $+1700$

$-1300$

(GX-0634, unpub.) on mammoth tusk from same deposit (Sissons, 1967).

Birm-165. Ballymakegoge, Co. Kerry, Ireland

$>42,500$

Laminated peat exposed below high tide level at Ballymakegoge, near Tralee, Co. Kerry, Ireland (52 $16^{\prime} \mathrm{N}$ Lat, $9^{\circ} 48^{\prime} \mathrm{W}$ Long). Coll. 1969 and subm. by G. F. Mitchell. Comment: determination supports Mitchell's interpretation as Hoxnian.

Birm-166. Derryvree, Co. Fermanagh, Ireland $\mathbf{3 0 , 5 0 0}$ $-1030$

Plant debris in laminated sand lens at $3.5 \mathrm{~m}$ depth between upper and lower tills (upper in drumlin form) at Derryvree, near Maguire's Bridge, Co. Fermanagh, Ireland $\left(54^{\circ} 18^{\prime} \mathrm{N}\right.$ Lat, $7^{\circ} 27^{\prime} \mathrm{W}$ Long, Grid Ref. H361390). Coll. 1969 by E. Colhoun; subm. by G. F. Mitchell. Comment: 2 tills are separated by interstadial deposits of Upton Warren date containing cold climate plants and beetles consistent with this dating.

\section{BRITISH LATE-GLACIAL AND HOLOGENE}

\section{Church Stretton series, Shropshire}

Samples from borehole near sewer manhole $\mathrm{MH} 60$ at Church Stretton, Shropshire (52 $32^{\prime} 30^{\prime \prime} \mathrm{N}$ Lat, $2^{\circ} 48^{\prime} 10^{\prime \prime} \mathrm{W}$ Long, Grid Ref. SO456941). From $1.65 \mathrm{~m}$ clay, silt, and peat, underlying $1.3 \mathrm{~m}$ solifluction gravel and overlying $1.32 \mathrm{~m}+$ pebbly clay and gravel upon till. Coll. 1967 by P. J. Osborne; subm. by F. W. Shotton.

\section{Birm-148.}

$11,000 \pm 200$

9050 B.C.

Plant fragments from gray clay, 0 to $0.2 \mathrm{~m}$ below solifluction gravel.

\section{Birm-158.}

$12,135 \pm 200$

Plant fragments from peat between 1.02 and $1.29 \mathrm{~m}$ below solifluction gravel.

\section{Birm-149.}

$13,555 \pm 620$

\section{1,605 в.c.}

Plant fragments washed from gray clay between 1.29 and $1.45 \mathrm{~m}$ below solifluction gravel. Sample small, hence high standard deviation. General Comment: dates confirm evidence (plants and coleoptera) that sequence covers Zones II and I, setting limiting dates to overlying solifluction gravels and underlying till. Birm-148 differs appreciably from NPL81 (11,790 \pm 140 , Callow, Baker, and Hassall, 1965) which refers to an intermediate between 148 and 158 horizon in adjacent trench sec. 


\section{Birm-92. Rodbaston Hall, Staffordshire}

Peat from core ca. $2 \mathrm{~m}$ depth in borehole, Rodbaston Hall, Stafford-

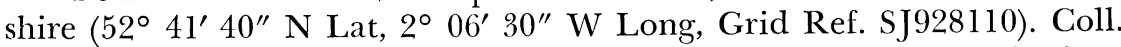
1966 by C. H. S. Sands; subm. by A. C. Ashworth. Sample from horizon where extreme $\mathrm{N}$ coleoptera disappeared from faunal spectrum.

\section{Birm-118. Penkridge, Staffordshire}

$11,580 \pm 140$

Plant material from sandy peat at $2.8 \mathrm{~m}$ depth in gravels overlying Keuper Sandstone at Penkridge, Staffordshire $\left(52^{\circ} 43^{\prime} 35^{\prime \prime} \mathrm{N}\right.$ Lat, $2^{\circ} 06^{\prime}$ 45" W Long, Grid Ref. SJ924143). Coll. 1968 and subm. by A. V. Morgan.

Birm-131. Pillaton Hall, Staffordshire

$11,660 \pm 250$ 9710 B.C.

Plant material from base of sandy peat overlying sand at ca. $3 \mathrm{~m}$ depth in peat bog, at Pillaton Hall near Penkridge, Staffordshire $\left(52^{\circ}\right.$

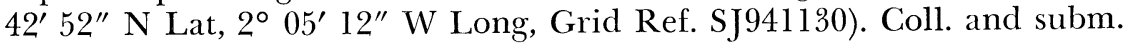
by A. V. Morgan. Comment: dates beginning of organic filling of hollow of kettle form.

\section{Birm-150. Borehole 12, Stafford}

$13,490 \pm 380$

11,540 в.c.

Plant fragments from dark gray silt at $15.6 \mathrm{~m}$ depth in Borehole 12 of Inner Relief Rd., Stafford (52 $48^{\prime} 24^{\prime \prime} \mathrm{N}$ Lat, 2 ${ }^{\circ} 06^{\prime} 30^{\prime \prime} \mathrm{W}$ Long, Grid Ref. SJ927233). Coll. 1969 and subm. by A. V. Morgan. Comment: dates base of unusually thick peaty silts resting on $9 \mathrm{~m}$ fluvioglacial deposits.

\section{Birm-135. Fladbury, Worcestershire}

$9030 \pm 200$ $\mathbf{7 0 8 0}$ B.c.

Roots (probably sedges) from silty peat beneath $1.5 \mathrm{~m}$ red clay-sand and above $4 \mathrm{~m}$ gravel of Avon No. 1 terrace at Fladbury Lower Moor, Worcestershire (52 $06^{\prime} 45^{\prime \prime} \mathrm{N}$ Lat, $2^{\circ} 01^{\prime} 45^{\prime \prime} \mathrm{W}$ Long, Grid Ref. SO 981461). Coll. 1969 by P. Buckland; subm. by F. W. Shotton. Comment: 1st date from this terrace, lowest of Avon series.

\section{Birm-153. Bransford, Worcestershire}

$2060 \pm 170$

Wood imbedded at $5.1 \mathrm{~m}$ depth in alluvial gravel of $\mathrm{R}$. Teme with remains of Cervus elaphus, at New House Farm, Bransford, Worcestershire (52 $12^{\prime} 30^{\prime \prime} \mathrm{N}$ Lat, $2^{\circ} 18^{\prime} \mathrm{W}$ Long, Grid Ref. SO798533). Coll. 1969 and subm. by G. R. Coope.

\section{Birm-82. Orleton, Herefordshire}

$11,730 \pm 770$ 9780 B.c.

Moss fragments hand picked from laminated calcareous silt lens in outwash gravels of Wye glacier at Orleton, Herefordshire $\left(52^{\circ} 18^{\prime} 20^{\prime \prime}\right.$ 
N Lat, $2^{\circ} 44^{\prime} 30^{\prime \prime}$ W Long, Grid Ref. SO497677). Coll. 1967 by P. Cross; subm. by G. R. Coope. Comment: no alkali pretreatment because sample small. Modern roots known to penetrate sample so no guarantee that all contamination removed. Date older than previous determination of bulk sample $(5020 \pm 130)$ but must be regarded as minimal age only.

\section{Birm-105. Northmoor, Oxfordshire}

$11,250 \pm 100$

Peat from silt lens containing coleoptera ca. $1.5 \mathrm{~m}$ depth in terrace gravel at Brown's Pit ca. $1.2 \mathrm{~m}$ NNW of church, Northmoor, Oxfordshire (51 $44^{\prime} 00^{\prime \prime}$ N Lat, $1^{\circ} 23^{\prime} 35^{\prime \prime}$ W Long, Grid Ref. SP419041). Coll. 1968 by H. P. Powell; subm. by J. M. Edmonds.

\section{Birm-123. Rockingham, Northamptonshire}

$2170 \pm 280$

Charcoal fragments from old soil B horizon disturbed by slipped mass of Upper Lias clay at Gretton Wood, Rockingham, Northamptonshire (52 $31^{\prime} \mathrm{N}$ Lat, $0^{\circ} 41^{\prime} \mathrm{W}$ Long, Grid Ref. SP883923). Coll. 1968 and subm. by R. J. Chandler. Comment: provides lower limit to date of landslip.

\section{Birm-106. Oaze Deep, River Thames}

$11,900 \pm 540$

9950 в.c.

Shells (mainly Cardium and Mytilus) in laminated silty clay from core at $-19 \mathrm{~m}$ alt, $6.7 \mathrm{~m}$ below bed of Thames Estuary at Oaze Deep $\left(51^{\circ} 32^{\prime} 25^{\prime \prime} \mathrm{N}\right.$ Lat, $1^{\circ} 08^{\prime} 10^{\prime \prime}$ E Long). Coll. 1966 by George Wimpey and Co.; subm. by R. J. Maddrell. Comment: because of small sample, measurement made on whole sample.

\section{Birm-167. Lewes Brooks, Sussex}

$5670 \pm 170$

Plant fragments from silty peat between 6.7 and $6.9 \mathrm{~m}$ depth (ca. $-4 \mathrm{~m}$ alt) in Borehole B 117 at Lewes Brooks, Lower Ouse Valley, Sussex $\left(50^{\circ} 52^{\prime} \mathrm{N}\right.$ Lat, $0^{\circ} 0^{\prime}$ Long, Grid Ref. TQ413092). Coll. 1969 and subm. by $\mathrm{A}$. Thorley and $\mathrm{D}$. K. Jones.

\section{Birm-168. Lewes Brooks, Sussex}

$6290 \pm 180$ 4340 в.c.

Plant material from silty peat at 9.5 to $9.8 \mathrm{~m}$ depth (ca. $-5.6 \mathrm{~m}$ alt) underlying silt, in Borehole B 123 at Lewes Brooks, Lower Ouse Valley, Sussex $\left(50^{\circ} 42^{\prime} \mathrm{N}\right.$ Lat, $0^{\circ} 0^{\prime}$ Long, Grid Ref. TQ413013). Coll. 1969 and subm. by A. Thorley and D. K. Jones. Comment: with Birm167 dates events in Holocene vegetational history of SE England and provides limiting dates to marine transgression in Lower Ouse Valley.

\section{Red Moss series, Lancashire}

Peat samples from borehole at Red Moss, near Horwich, Lancashire (53 35' 23" N Lat, 2० 34' 36" W Long, Grid Ref. SD632102). Coll. 1968 and subm. by A. C. Ashworth. 


\section{(a) 9800 \\ (b) $8390 \pm 100$ 6440 B.c.}

Birm-124.

Sample after alkali pretreatment (a) and humate extract (b) from base of woody peat layer above gray silty clay.

\section{Birm-128.}

$10,850 \pm 120$ 8900 B.C.

Sample from top of peat layer, immediately underlying gray silty clay, $0.3 \mathrm{~m}$ below Birm-124.

\section{Birm-127.}

$$
12,160 \pm 140
$$

Sample from base of peat layer $0.35 \mathrm{~m}$ below gray silty clay and $0.55 \mathrm{~m}$ below sample Birm-124.

General Comment: sec. contains coleopterous fauna studied by A.C.A. Fauna of Birm-127 does not indicate cold climate, Birm-128 marks incoming of cold species, and Birm-124 dates disappearance of arctic stenotherms.

\section{Heysham series, Lancashire}

Plant material from sedge peat beneath marine clay and sand, overlying sand and boulder clay in offshore boreholes drilled 1967 near Heysham, Lancashire ( $54^{\circ} 02^{\prime} \mathrm{N}$ Lat, $2^{\circ} 56^{\prime} \mathrm{W}$ Long). Coll. 1968 by A. Ashworth; subm. by F. W. Shotton.

\section{Birm-139. Borehole M1}

$9195 \pm 155$

Sample at $-16.4 \mathrm{~m}$ alt, Grid Ref. SD395599.

7245 в.c.

\section{Birm-140. Borehole M2}

$8925 \pm 200$

Sample from -15.8 to $-16.3 \mathrm{~m}$ alt, Grid Ref. SD394599.

\section{Birm-141. Borehole M3}

$9270 \pm 200$

Sample from -17.6 m alt, Grid Ref. SD393599. 7320 в.C.

General Comment: series gives evidence for Post Glacial rise of sea level in Morecambe Bay.

\section{Birm-147. Holcombe Moor, Lancashire}

$3540 \pm 120$ 1590 B.c.

Twigs (Betula) at $0.9 \mathrm{~m}$ depth in $0.25 \mathrm{~m}$ thick basal layer of peat bog at Holcombe Moor, Lancashire (53 $38^{\prime} \mathrm{N}$ Lat, $2^{\circ} 20^{\prime} \mathrm{W}$ Long, Grid 
Ref. SD777169). Coll. 1969 and subm. by J. H. Tallis. Comment: sample helps give time scale for moorland peat accumulation.

\section{Birm-120. Greenock, Renfrewshire}

$9890 \pm 160$

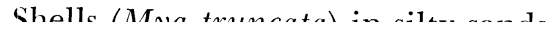

7940 B.C. 


\section{Sorgfjord series, Vestspitsbergen}

Samples coll. in Sorgfjord region, Vestspitsbergen to help give rate 
sec. on N side Agua de Pau volcano $1.5 \mathrm{~km} \mathrm{NE}$ of Lombadas, Sao Miguel, Azores (37 $47^{\prime}$ N Lat, $25^{\circ} 27^{\prime}$ W Long). Coll. 1968 and subm. by G. P. L. Walker.

\section{Birm-126. Faial, Azores}

$1200 \pm 70$

Charcoal from 2nd from top of 11 ash beds from summit caldera of Faial, Azores, exposed in rd. sec. $2.5 \mathrm{~km} \mathrm{~N}$ of edge of caldera $\left(38^{\circ} 36^{\prime}\right.$ $30^{\prime \prime}$ N Lat, $28^{\circ} 42^{\prime} 30^{\prime \prime}$ W Long). Coll. 1968 and subm. by G. P. L. Walker.

\section{Birm-156. Tuitts' Ghaut, Montserrat, W Indies}

$18,390 \pm 360$

Charcoal from base of ca. $50 \mathrm{~m}$ thick pumice flow believed assoc. with formation of English's Crater and in upper part of Soufriere Hills pyroclast flow succession at Tuitts' Ghaut, Montserrat, W Indies $\left(16^{\circ}\right.$

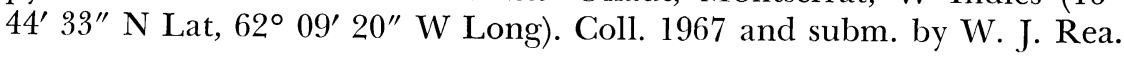

\section{7,900 \\ Birm-115. King Point, Yukon, Canada \\ 35,950 в.c. \\ $+2800$}

Wood at $+8 \mathrm{~m}$ alt imbedded in $25 \mathrm{~m}$ thick unconsolidated sand and silt underlying sand and gravel exposed in vertical coastal cliff $1.6 \mathrm{~km} \mathrm{~W}$ of King Point, Yukon, Canada $\left(69^{\circ} 07^{\prime} \mathrm{N}\right.$ Lat, $138^{\circ} 01^{\prime} \mathrm{W}$ Long, Grid Ref. 117A/East). Coll. 1968 by D. McIntyre; subm. by D. Naylor. Comment: overlying gravel, dated at 6000 (unpub.), lies unconformably (Naylor, unpub.) or is overthrust (Mackay, 1959) upon earlier sediments here dated.

\section{Birm-96. Monte Amargo, Chile}

$\mathbf{8 8 0} \pm 120$

Collagen fraction from bone of medium-size herbivore in dry marsh on S bank R. Copiapó, near Monte Amargo, Chile $\left(27^{\circ} 22^{\prime} \mathrm{S}\right.$ Lat, $70^{\circ}$ 43' W Long). Coll. 1967 and subm. by C. Mortimer. Comment: dates a time in pluvial period that preceded desiccation of low-level terrace of Rio Copiapó.

\section{Birm-17. Marian Cove, King George Island}

$1430 \pm 470$

Seaweed from ca. $2.7 \mathrm{~m}$ depth in bedded gravels underlying raised beach at $+5 \mathrm{~m}$ alt $\mathrm{E}$ of South Spit $\mathrm{S}$ shore Marian Cove, King George I., Antarctica (62 $14^{\prime} \mathrm{S}$ Lat, $58^{\circ} 48^{\prime} \mathrm{W}$ Long). Coll. 1966 by D. E. Sugden; subm. by B. S. John. Comment: sample should be older than modern seaweed, Birm-16 $1223 \pm 81$ (Shotton, Blundell, and Williams, 1968, p. 203) but result inconclusive. Large error due to small sample. 
Birm-145. Tongariro, North Island, New Zealand
$\mathbf{2 6 0 0} \pm \mathbf{6 5 0}$ B.c.
Carbonized branch imbedded in Wanganui pumice gravel S side rd. sec. State Hwy. 47 at Tongariro, North I., New Zealand (39 $9^{\prime} 40^{\prime \prime}$ S Lat, $175^{\circ} 35^{\prime}$ E Long). Coll. 1969 and subm. by C. A. Fleming. Comment: duplicate sample sent to Inst. Nuclear Sci., New Zealand, for dating. Confirms that this ash shower antedates Taupo Ash (Healy, Vucetich, and Pullar, 1964).

IV. ARCHAEOLOGIC SAMPLES

\section{A. British}

Birm-58. Wadden Hill, Dorset

$2140 \pm 180$

190 B.C.

Charcoal from ca. $1.5 \mathrm{~m}$ depth in pit at Roman Fort occupied A.D. 45 to 60 (Webster, 1965) at Wadden Hill near Stoke Abbott, Dorset $\left(50^{\circ} 48^{\prime} \mathrm{N}\right.$ Lat, $2^{\circ} 47^{\prime} \mathrm{W}$ Long, Grid Ref. 450015). Coll. 1968 and subm. by G. Webster. Comment: indicates problem of dating charcoal when it may be derived from wood of old trees.

\section{Birm-109. Tamworth, Staffordshire}

$1541 \pm 80$

Oak plank ca. $4 \mathrm{~m}$ deep in filling of main Saxon defensive ditch of Tamworth (52 $38^{\prime} \mathrm{N}$ Lat, $1^{\circ} 42^{\prime} \mathrm{W}$ Long, Grid Ref. SK206038). Coll. 1968 by C. S. Young; subm. by P. A. Rahtz. Comment: maximum date, since plank probably comes from timber structure assoc. with defenses.

\section{Hereford series}

Charcoal samples from excavations at Hereford $\left(52^{\circ} 04^{\prime} \mathrm{N}\right.$ Lat, $2^{\circ} 44^{\prime} \mathrm{W}$ Long, Grid Ref. SO508404). Coll. 1968 and subm. by P. A. Rahtz.

Birm-111.

$$
1189 \pm 83
$$

A.D. 761

Charcoal from pit of corn-drying oven beneath rampart of Birm-110.

\section{Birm-110.}

$1335 \pm 67$

Charcoal residue of large structural timbers at ca. $2 \mathrm{~m}$ depth in major defensive rampart of Saxon town.

Birm-159.

(a) $700 \pm 220$

A.D. 1250

(b) $1330 \pm 200$

A.D. 620

Sample after alkali pretreatment (a) and humate extract (b) of charcoal residue from large structural timbers at ca. $1 \mathrm{~m}$ depth in major defensive rampart. 


\section{Birm-112. Metchley Camp, Birmingham}

A.D. 1661

$289 \pm 79$

Brushwood at ca. $0.7 \mathrm{~m}$ depth at base of trench which cuts all Roman structures at Metchley Camp, Birmingham (52 $27^{\prime} 0^{\prime \prime} \mathrm{N}$ Lat, $1^{\circ} 56^{\prime} 20^{\prime \prime}$ W Long, Grid Ref. SP042836). Coll. 1968 and subm. by T. Rowley. Comment: last trench cutting complex of Roman structures, hopefully dating end of Roman occupation, but proving to be recent.

\section{Birm-119. South Barrule, Isle of Man}

$2473 \pm 84$

523 в.c.

Charcoal assoc. with pottery, from upper hearth level of hut in hill-fort (Gelling, 1963) on South Barrule, Isle of Man $\left(54^{\circ} 09^{\prime} \mathrm{N}\right.$ Lat, $4^{\circ} 40^{\prime}$ W Long, Grid Ref. SC258759). Coll. 1968 and subm. by P. S. Gelling. Comment: proof of early Iron age.

\section{Birm-129. Dorstone, Herefordshire}

$1910 \pm 90$

Charcoal from supposed Neolithic hearth cut by post hole. Later Roman-British occupation of site, Dorstone Hill, Herefordshire $\left(52^{\circ} 04^{\prime}\right.$ N Lat, $2^{\circ} 59^{\prime}$ W Long, Grid Ref. SO326423). Coll. 1968 and subm. by W. R. Pye. Comment: hearth is part of Romano-British complex.

\section{Birm-130. Rowington, Warwickshire}

$1850 \pm 110$

Charcoal from $1.5 \mathrm{~m}$ depth in stake hole of Roman tile kiln at Rowington, Warwickshire $\left(52^{\circ}, 19^{\prime} 30^{\prime \prime} \mathrm{N}\right.$ Lat, 1 $1^{\circ} 43^{\prime} 30^{\prime \prime}$ W Long, Grid 


\section{Midsummer Camp series, Herefordshire}

Samples assoc. with successive building of 17 gates throughout long period of defense of hill fort, Midsummer Camp, Eastnor, Herefordshire $\left(52^{\circ} 02^{\prime} \mathrm{N}\right.$ Lat, $2^{\circ} 21^{\prime} \mathrm{W}$ Long, Grid Ref. SO761374). Coll. 1967 and subm. by S. C. Stanford.

\section{Birm-142.}

$2370 \pm 190$

Wood from quarry ditch floor at $1 \mathrm{~m}$ depth, $\mathrm{W}$ of $\mathrm{S}$ gateway, assoc. with 1st gate.

\section{Birm-143.}

$2000 \pm 100$

Carbonized grain at $1 \mathrm{~m}$ depth, E side $\mathrm{S}$ gateway, assoc. with destruction of 8 th gate.

\section{Birm-144. Croft Ambrey, Aymestry, Herefordshire}

$3000 \pm 200$

Carbonized grain from $1 \mathrm{~m}$ depth in quarry-ditch behind main rampart of Croft Ambrey Hill Fort, Aymestry, Herefordshire $\left(52^{\circ}{ }^{1} 8^{\prime}\right.$ N Lat, $2^{\circ} 49^{\prime} \mathrm{W}$ Long, Grid Ref. SO445668). Coll. 1962 and subm. by S. C. Stanford. Comment: date anomalously old. 
Birm-103. Chiozza

Collagen fraction of bone (Bos) in pit assoc. with Chiozza phase at Monte Rocca, Rivoli, Italy (46 $06^{\circ} \mathrm{N}$ Lat, $10^{\circ} 50^{\prime} \mathrm{E}$ Long). Coll. 1967 by L. H. Barfield.

\section{Birm-104. Rivoli Rocea}

$5670 \pm 130$

Collagen fraction of mixed bone (mainly Bos and Sus) from storage pit assoc. with Rivoli Rocca phase, Monte Rocca, Rivoli, Italy $\left(45^{\circ} 50^{\prime}\right.$ N Lat, $10^{\circ} 50^{\prime}$ E Long). Coll. 1967 by L. H. Barfield.

\section{Molino Casarotto series, Italy}

Charcoal and wood samples from site of early Neolithic occupation at Molino Casarotto, Arcugnano, Vicenza, Italy (45 $28^{\prime} \mathrm{N}$ Lat, $11^{\circ} 36^{\prime}$ E Long). Coll. 1969 and subm. by L. H. Barfield. Nine other samples from site subm. to Rome for radiocarbon dating.

\section{Birm-172. Sample 10}

$6240 \pm 100$

Charcoal fragments in body of shell midden lying on lake marl, below peat and ca. $0.5 \mathrm{~m}$ thick clay, in Sqs. $38 \mathrm{~N}, \mathrm{O}$ and $\mathrm{P}$, Site 4.

Birm-173. Sample 11

$6290 \pm 150$

Charcoal fragments contained in shell midden lying on marl and below peat in Sq. 41A, Site 4 .

\section{Birm-174. Sample 12}

$6350 \pm 140$

Charcoal from bottom horizon of multilevel hearth in center of wooden house, Sq. 38L, Site 4 .

\section{Birm-175. Sample 13}

$6450 \pm 110$

Wood from 3rd layer of cross set timbers in platform, preserved in peat below ca. $0.5 \mathrm{~m}$ clay, assoc. with Neolithic artifacts, from Trench 2, Site 3 .

\section{Birm-176. Sample 14}

$6470 \pm 150$

Wood from beam forming part of substructure of wooden house, underlying hearth of Birm-174, from Sq. 37K, Site 4 .

\section{Birm-177. Sample 15}

$6125 \pm 150$

4175 в.c.

Peat from deposit surrounding hearth and wooden house, belonging to final phase of settlement, from Level 3, Site 4. 
Birm-107. Apliki Mine, Cyprus

Wood (Pinus brutia) saturated in sulphide copper ore at $+291 \mathrm{~m}$ alt from Apliki Open Pit $4 \mathrm{~km} \mathrm{~S}$ of Lefka, Cyprus $\left(34^{\circ} 00^{\prime} \mathrm{N}\right.$ Lat, $32^{\circ}$ 20' E Long). Coll. 1967 and subm. by M. J. Bishop. Comment: date confirms antiquity of mines.

\section{Birm-116. Gressvannet, Nordland, Norway}

$3090 \pm 180$

Charcoal assoc. with quartzite arrowheads, of Younger Stone age culture from base of peat deposit, Gressvannet, Nordland, Norway $\left(66^{\circ}\right.$ 03' N Lat, $14^{\circ} 30^{\prime}$ E Long). Coll. 1968 and subm. by D. P. S. Peacock.

\section{Birm-117. Gressvannet, Nordland, Norway}

$6990 \pm 120$

Charcoal assoc. with crude scrapers of older stone age culture in sandy soil underlying peat bed containing sample Birm-116, Gressvannet, Nordland, Norway (66 $03^{\circ} \mathrm{N}$ Lat, 14 ${ }^{\circ} 30^{\prime} \mathrm{E}$ Long). Coll. 1968 and subm. by D. P. S. Peacock.

\section{Birm-154. Dumpo Quarter, Brong/Ahafo, Ghana A.D. 1243}

$$
707 \pm 92
$$

Charcoal ca. $0.8 \mathrm{~m}$ deep at top of Spit 4 in occupation mound at Dumpo Quarter, Brong/Ahafo, Ghana $\left(7^{\circ} 56^{\prime} 30^{\prime \prime} \mathrm{N}\right.$ Lat, $2^{\circ} 26^{\prime} 0^{\prime \prime} \mathrm{W}$ Long). Coll. 1967 and subm. by R. D. Mathewson.

\section{Birm-155. Dumpo Quarter, Brong/Ahafo, Ghana A.D. 1700}

$$
250 \pm 150
$$

Charcoal from ca. $0.5 \mathrm{~m}$ depth at base of latest burial level in Spit 2 of occupation mound at Dumpo Quarter, Brong/Ahafo, Ghana $\left(7^{\circ} 56^{\prime}\right.$ 30" N Lat, 2० 26' $0^{\prime \prime}$ W Long). Coll. 1967 and subm. by R. D. Mathewson. Comment: this and Birm-154 continue series started by Birm-71, 79, and 80 (Shotton, Blundell, and Williams, 1969, p. 269). Results inconsistent with earlier dates, as both underlie Birm-71, $931 \pm 158$. Both samples alkali pretreated and figures suggest disturbed stratigraphy.

\section{REFERENCES}

Callow, W. J., Baker, M. J., and Hassall, G. I., 1965, National Physical Laboratory radiocarbon measurements III: Radiocarbon, v. 7, p. 156-161.

Delibrias, G., Guillier, M. T., and Labeyrie, J., 1969, Gif natural radiocarbon measurements III: Radiocarbon, v. 11, p. 327-344.

Delibrias, G. and Larsonneur, C., 1966, Datation absolue de dépôts organiques würmiens en Normandie: Acad. sci. [Paris] Comptes rendus, v. 263, p. 1022-1024.

Evans, W. B., 1968, Geology of the country around Macclesfield, Congleton, Crewe and Middlewich: Geol. Survey Great Britain Mem., no. 110.

Gelling, P. S., 1963, Excavations at the hill-fort on S Barrule: Isle of Man Nat. Hist. and Antiq. Soc. Proc., v. 6, p. 313-323.

Gregory, J. W. and Currie, E. D., 1928, The vertebrate fossils from the glacial and associated post-glacial beds of Scotland in the Hunterian Museum, Univ. of Glasgow: Geol. Dept. Hunterian Mus. Mon., Glasgow Univ., v. 2.

Healy, J., Vucetich, C. G., and Pullar, W. A., 1964, Stratigraphy and chronology of Late-Quaternary volcanic ash in Taupo, Rotorua, and Gisbourne districts: New Zealand Geol. Surv. Bull., no. 73, p. 34. 
Mackay, J. R., 1959, Glacier ice-thrust features of the Yukon coast: Geog. Bull., v. 13 , p. $5-21$.

Shotton, F. W., Blundell, D. J., and Williams, R. E. G., 1968, Birmingham University radiocarbon dates II: Radiocarbon, v. 10, p. 200-206. p. $263-270$.

Simpson, I. M. and West, R. G., 1958, On the stratigraphy and palaeobotany of a Late-Pleistocene organic deposit at Chelford, Cheshire: New Phytologist, v. 57, p. $239-250$.

Sissons, J. B., 1967, Glacial stages and radiocarbon dates in Scotland: Scottish J. Geol., v. 3, p. 375-381.

Vogel, J. C. and Zagwijn, W. H., 1967, Groningen radiocarbon dates VI: Radiocarbon, v. 9, p. 63-106.

Webster, G., 1965, Further investigations on the site of the Roman fort at Wadden Hill, Stoke Abbott, 1960 to 1962: Dorset Nat. Hist. and Archaeol. Soc. Proc., v. 86, p. $135-149$.

Worsley, P., 1967, Problems in naming the Pleistocene deposits of the North-East Cheshire Plain: Mercian Geologist, v. 2, p. 51-55. 